\title{
Reliable Wireless Communication Networks for Demand Response Control
}

\author{
Lei Zheng, Student Member, IEEE, Ning Lu, Senior Member, IEEE, and Lin Cai, Senior Member, IEEE
}

\begin{abstract}
This paper presents methodologies for deriving reliability performance of wireless communication networks to support demand response (DR) control. First, the impact of communication impairments on a direct $D R$ control program is investigated. Second, the outage probability of a wireless link is modelled and quantified, considering the multipath fading, shadowing, and random path loss given the location distribution of smart meters. Third, the distributions of packet delivery ratio are derived for two wireless network architectures: the single-hop infrastructure-based network and the multi-hop mesh network. Simulation results verify the above reliability models and provide important insights on the coverage of wireless communication networks considering the reliability requirements of DR programs.
\end{abstract}

Index Terms-Communications reliability, demand response, network topology, outage probability, smart grid.

\section{INTRODUCTION}

$\mathbf{T}$ HE bi-directional communication networking of the smart grid infrastructure enables many demand response (DR) technologies, which control hundreds or thousands of distributed energy resources over vast geographic areas [1]-[7]. Among access technologies, wireless communication networking is a promising solution because of low cost and wide coverage. However, it is critical to understand the reliability of wireless communications and to quantify its impact on DR performance, especially on DR programs that require frequent information exchange between the controller and end devices [1]. An example of such DR programs is the use of water heaters [2], [3] or heating, ventilation, and air conditioning (HVAC) units [4] for ancillary services. Assuming that each end device is controlled through a smart meter that relays the end device status to and receives control commands from the DR controller, the reliability of the wireless communication networks affects both the correctness of the controller decision process and the effectiveness of control performance.

Previous studies have revealed the considerable potential and benefits of DR programs. However, to ensure effective control performance, the impact of communication reliability on DR control must be addressed. In [8], the frequency with which information can be retrieved from and delivered to loads was in-

Manuscript received April 02, 2012; revised September 01, 2012; accepted October 02, 2012. Date of publication February 06, 2013; date of current version February 27, 2013. Paper no. TSG-00189-2012.

L. Zheng and L. Cai are with the Department of Electrical and Computer Engineering, University of Victoria, Victoria, BC V8P 5C2, Canada (e-mail: zhengl@ece.uvic.ca; cai@ece.uvic.ca).

N. Lu is with the North Carolina State University, Raleigh, NC 27695 USA (e-mail:nlu2@ncsu.edu).

Digital Object Identifier 10.1109/TSG.2012.2224892 vestigated but other communication impairments such as packet losses were ignored. In [9], a discrete Markov chain model was adopted to quantify the packet losses due to the buffer-overflow at the data aggregator (DA), but the impact of wireless communication errors between the smart meters and the DA was not considered. For a general wireless network, [10], [11] studied the communication reliability using Bernoulli processes with parameter $p$. However, a method for obtaining $p$ has not been addressed. In [12], the reliability of a multi-hop wireless communication system and its impact on DR was studied using Monte Carlo simulations.

This paper focuses on reliability analysis of wireless access networks for DR applications. The main contributions of this work are threefold. First, we evaluate and compare the impact of wireless communication errors on the DR control strategy in two cases. In the first case, it is assumed that packet losses randomly occur to all devices; while in the second case, the communication losses are concentrated within a certain group of users who experience worse communication conditions than other users. Second, we use the outage probability as the performance metric to analyze the reliability of communication services in the smart grid. To quantify the outage probability of wireless communication links, three aspects of random effects are considered: i) the log-normal shadowing effect, ii) Rayleigh fading, and iii) the random locations of smart meters. Numerical approximations to link outage probability are derived. Given the analytical model of link reliability, the reliability in both single-hop and multi-hop wireless networks is modeled and analyzed using binomial distribution and conditional binomial distribution. Finally, model accuracy is verified by comparing analytical and simulation results. In addition, this paper provides important insights on the coverage of wireless communication networks considering the reliability requirements of DR control.

The rest of this paper is organized as follows. Section II describes the smart grid system model and discusses the main factors affecting wireless communication reliability. In Section III, we present a sample DR control strategy [4], and evaluate the impact of communication impairments on demand response. The model of wireless communication link reliability is presented in Section IV. In Section V, the reliability of wireless communication networks is studied, followed by model validations in Section VI. We conclude the paper and discuss the further research issues in Section VII.

\section{SYSTEM MODELS}

In this paper, we assume that houses equipped with smart meters are distributed within a service area with one central DA to collect power-related information from meters and to deliver 
TABLE I

NotATIONS USED IN THIS PAPER

\begin{tabular}{|c|c|}
\hline Notation & Explanation \\
\hline$x, y$ & the transmitted and received signal, \\
\hline$g$ & the channel power gain, \\
\hline$n, N_{0}$ & the white Gaussian noise and its power \\
\hline$l,\left[L^{-}, L^{+}\right]$ & the communication distance and its scope, \\
\hline$p l, s$ & the path-loss and shadowing effect, \\
\hline$m, M$ & the number of hops along a routing path \\
\hline$P_{t}$ & the transmitting power, \\
\hline$\epsilon, K$ & the path-loss component and constant \\
\hline & depending on carrier frequency and antenna gain, \\
\hline$\sigma$ & the std of shadowing effect, \\
\hline$\mu_{a}, \sigma_{a}$ & the equivalent mean and std in approximated \\
\hline & SNR distribution, \\
\hline$C_{e}$ & the Euler's constant, \\
\hline$\eta$ & the constant in log-normal shadowing effect, \\
\hline$\gamma, \Gamma$ & $\begin{array}{l}\text { the signal-to-noise ratio (SNR) and the threshold, } \\
\text { less than which link outage happens, }\end{array}$ \\
\hline$N$ & the order of Legendre/Hermite polynomial, \\
\hline$x_{i}^{g l}, w_{i}^{g l}$ & the root of Legendre polynomial and its weight, \\
\hline$x_{i}^{g h}, w_{i}^{g h}$ & $\begin{array}{l}\text { the root of Hermite polynomial and its weight, } \\
\text { the ratio of packets successfully delivered, }\end{array}$ \\
\hline$R, E$ & $\begin{array}{l}\text { the edge length of a square cluster } \\
\text { and the square coverage area }\end{array}$ \\
\hline$f_{S}(s \mid l), f_{G}(g \mid s)$ & $\begin{array}{l}\text { the number of smart meters in the coverage area, } \\
\text { the PDF of shadowing effect given communication } \\
\text { distance and channel gain given shadowing effect, }\end{array}$ \\
\hline$f_{\Gamma}(\gamma \mid l), f_{\Gamma}^{\prime}(\gamma \mid l)$ & $\begin{array}{l}\text { the PDF of SNR given communication distance, } \\
\text { and its approximation, }\end{array}$ \\
\hline$f_{L}(l)$ & the PDF of distance distribution, \\
\hline$P_{o}(\gamma \mid l)$ & the conditional link outage probability, \\
\hline $\begin{array}{c}P_{o}(\gamma), P_{o}^{(m)}(\gamma) \\
P_{h}(m) \\
P_{s}(\gamma) \\
\theta\end{array}$ & $\begin{array}{l}\text { the probability of link outage for one or } m \text { hop }(\mathrm{s}) \text {, } \\
\text { the probability of an } m \text { hops routing path, } \\
\text { the probability for a successful end-to-end delivery, } \\
\text { the packet delivery ratio, }\end{array}$ \\
\hline$P_{s}^{1 h}(\theta), P_{s}^{m h}(\theta)$ & $\begin{array}{l}\text { the probability that packet delivery ratio } \geq \theta \\
\text { in a single or multi-hop network, }\end{array}$ \\
\hline$\left[T^{-} \stackrel{\rho}{,} T^{+}\right]$ & $\begin{array}{l}\text { the assumed communication error ratio, } \\
\text { the comfort room temperature region. }\end{array}$ \\
\hline
\end{tabular}

control commands to those meters. Table I summarizes the notations used in this paper.

\section{A. Reliability Index}

We first define the wireless communication reliability index at different levels. For the reliability of a wireless link, link outage probability is used. For reliability at the network-level, which is composed of multiple links, reliability is evaluated by the packet delivery ratio. These two performance indexes are defined separately in Definitions 1 and 2.

Definition 1: Link outage probability is the probability that the link quality is insufficient to support communication requirements. In a lossy wireless communication network, a link is considered reliable if its outage probability is lower than a predefined threshold.

Definition 2: Given a number of packets to be transmitted, packet delivery ratio is defined as the ratio of the number of packets successfully received at the destination(s) over the number of packets transmitted.

Given the definition of reliability performance indexes, there are several common factors affecting the wireless communication reliability, including the network topology, the collision or

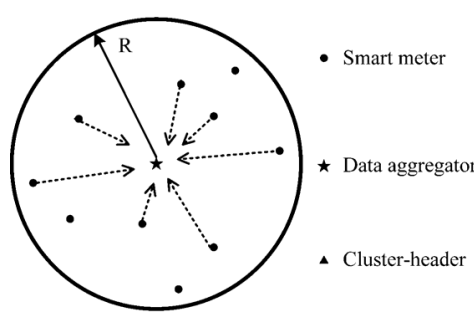

(a)

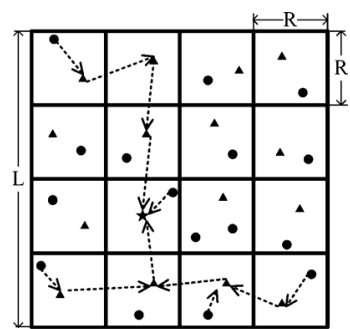

(b)
Fig. 1. Network topologies. (a) A single-hop network. (b) A multi-hop network.

buffer overflow in medium access control (MAC), and the probabilistic wireless channel behavior. Models and assumptions of these factors are presented as following.

\section{B. Network Topology and Routing}

Depending on the coverage area, an important issue is network topology design, which defines how to construct the wireless network (such as using a single-hop or a multi-hop architecture). For a wireless link, the longer the distance between the source and the destination, the higher the probability of packet error. If a relay is introduced, the transmission range of a single hop is reduced, but the number of hops increases, which makes the hop-by-hop transmission more complex and error prone.

In this paper, we consider both single-hop and multi-hop wireless access networks, as the two cases shown in Fig. 1. A single-hop wireless network covers a circular area, where information packets or control commands are directly delivered between the smart meters and the DA. For a multi-hop network, smart meters are distributed in a square area and organized into square-shape clusters with a cluster-header working as relay nodes, collecting data packets from its cluster members and forwarding these packets to the DA through other cluster-headers. Depending on the distance between adjacent cluster-headers, hop forwarding may occur multiple times, using the Manhattan Walk routing scheme [13] and the same routing path for bi-directional communications.

\section{MAC Protocol}

The contention-based MAC protocols are not desirable for applications with constant bit-rate traffic or requirements of high-reliability assurance, because packets can be dropped due to collisions in contention access. For DR in the smart grid, the requirements of communication resource is typically predictable, because most smart meters installed in houses are likely to be static and communication traffic is consistently low and periodic [1]. Considering these characteristics, we adopt a reservation-based MAC protocol using medium sharing schemes, such as time division multiple access (TDMA), and ignore packet losses due to buffer overflow as the traffic load for DR control is deterministic and low. Thus, the unreliable wireless communications studied in this paper are mainly due to the network topology and wireless channel behavior.

\section{Wireless Channel Model}

The wireless channel behavior has a significant influence on the packet delivery errors. To model a realistic wireless channel, 
the log-normal shadowing effect and Rayleigh fading are considered, assuming that the channel is static during a packet transmission. For a packet delivery, the signal that arrives at the destination is

$$
y=\sqrt{g} \cdot x+n,
$$

where $x$ is the transmitted signal, $n$ is the additive white Gaussian noise with variance $N_{0}$, and $g$ is the channel power gain, which is exponentially distributed with the mean varying independently according to a shadowing effects, and determined by the path-loss. For the path-loss, $p l=K l^{-\epsilon}$, where $l$ is the distance between the source and destination, $\epsilon$ is the path-loss component, and $K$ is a constant dependent on the carrier frequency and antenna gain. For the log-normal shadowing effect with given distance $l$, we have its probability density function $(\mathrm{PDF}), f_{S}(\cdot)$ as

$$
\begin{aligned}
f_{S}(s \mid l)= & \frac{\eta}{\sigma \sqrt{2 \pi} s} \\
& \cdot \exp \left\{\frac{-\left[10 \log _{10}(s)-10 \log _{10}\left(K l^{-\epsilon}\right)\right]^{2}}{2 \sigma^{2}}\right\},
\end{aligned}
$$

where $s$ is the shadowing effect, $\eta=\ln 10 / 10$ is a constant, and $\sigma$ is the standard variance of shadowing effect in decibels (dB). For the Rayleigh fading channel given the shadowing effect $s$, we have the PDF of channel power gain $f_{G}(\cdot)$ as

$$
f_{G}(g \mid s)=\frac{1}{s} e^{-g / s}
$$

The randomness of smart meter locations is also considered in this paper. Assuming smart meters are distributed as a Poisson point process in a specified region, the distance between a source and a destination becomes a random variable, which depends on the wireless communication network topology [14]. In the following, the PDF of random distance in a network is indicated as $f_{L}(\cdot)$.

\section{IMPACT OF COMMUNICATIONS ON DEMAND RESPONSE CONTROL STRATEGY}

In this section, the impact of wireless communications on the performance of the DR programs is simulated using the models and the direct DR control strategy proposed in [4]. From this simulation, the reliability requirements for the communication network are derived. Note that communication network problems have different impacts on different DR programs because the load models and control strategies may or may not be sensitive to communication delays or errors. A good DR controller design should account for realistic communication impairments.

\section{A. Demand Response Control Strategy}

In Section IV of [4], the performance of a temperature-priority-list-based direct load control scheme is used to aggregate $1000 \mathrm{HVAC}$ loads (with a temperature bandwidth of $4^{\circ} \mathrm{C}$ and an outdoor daily average temperature of $0^{\circ} \mathrm{C}$ ) for load balancing services. Two types of control signals are used: the regulation signal and the load following signal. Both control signals are normalized to $\pm 1 \mathrm{MW}$. As demonstrated in [4], if reliable and

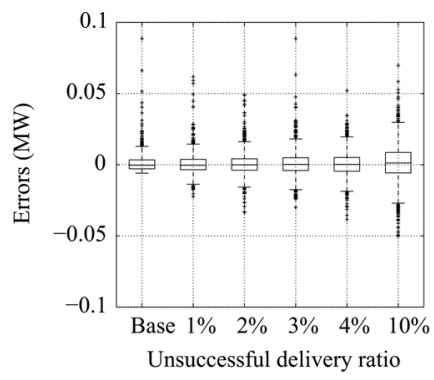

(a)

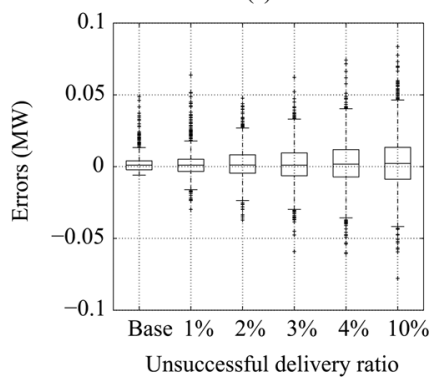

(c)

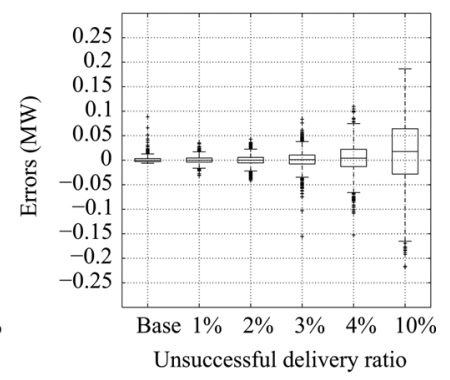

(b)

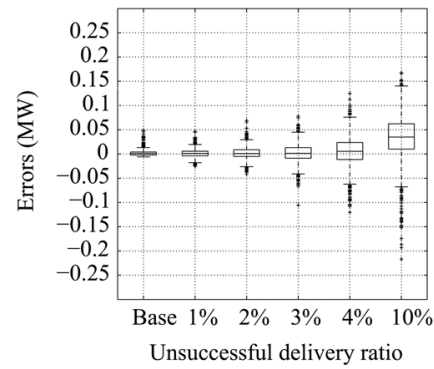

(d)
Fig. 2. Impact of communication errors on following and regulation signals. In the figure, the line in the middle of the box indicate the mean value of the control error samples, the boxes above and below the mean value represent the 25 th and 75 th percentiles of the samples respectively, and the points outside the boxes represent the samples beyond the $99.3 \%$ coverage if the data is normally distributed. (a) Load following case I. (b) Load following case II. (c) Regulation case I. (d) Regulation case II.

accurate bi-directional communications are always available, the performance meets load balancing requirements well.

\section{B. The Impact of Communication Errors}

To illustrate the impact of communication errors on the effectiveness of DR programs, we re-run the above simulations considering communication impairments in the delivery of control commands from the control center to the HVAC units. Assuming that $\rho$ percent $(\rho=0,1,2,3,4$, and 10) of the control commands delivered to the 1000 HVAC units are either incorrect or lost, two scenarios (Case I and Case II) are simulated with different patterns of communication errors: In Case I, the packet losses occur randomly in the 1000 HVAC units. In Case II, the packet losses occur randomly in the first 100 of the 1000 HVAC units. We assume that if an unit does not receive commands from a central controller unit, it will remain in its previous state until the maximum or minimum local temperature setting is validated. The control errors (the difference between the real power consumption and the targeted power consumption) are shown in Fig. 2. Violations of user comfort levels (shown in Fig. 3) are accounted for by calculating the amount of time in a day that room temperatures exceed the temperature region. The following observations are made from simulation results.

If the communication impairments occur randomly among 1000 HVAC units, DR performance is not significantly degraded. This is because, at each time interval, only a small percentage of HVAC units must be turned on or off. The probability of control commands not reaching those units can be small. For example, if 50 units need to switch from "on" to "off" and $\rho=4$, then on average only 2 units are expected to 


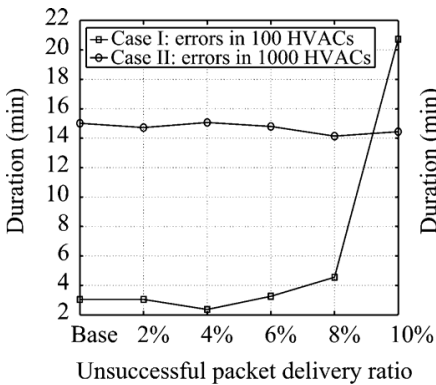

(a)

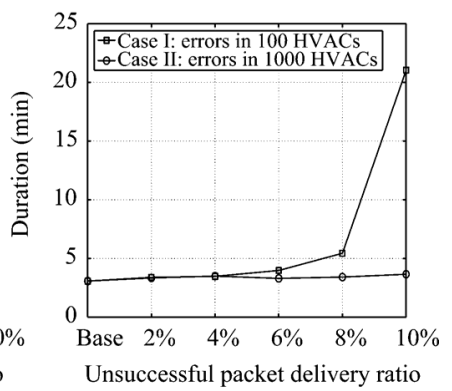

(b)
Fig. 3. Duration of comfort band violation. (a) Load following. (b) Regulation.

not respond. And the chance that these two units cannot receive a command in the following time interval is very low, which will not impact the overall performance significantly.

However, if the communication impairments occur only among certain HVAC units, the DR system performance can be significantly degraded. This is because at each time interval, $10 \rho$ percent of the 100 HVAC units will not follow the command. Cumulatively, some units may not receive a command for several time intervals, causing larger deviations from their targeted outputs.

User comfort is hardly affected, when the packet loss rate is less than $4 \%$. When the packet loss rate is greater than $4 \%$, there are times when room temperatures exceed the $\left[T^{-}, T^{+}\right]$region. The above analysis shows that it is critical to design communication networks so that the packet losses do not occur consistently within a small group of control objects and to ensure that packet losses do not exceed $10 \%$ to keep the control errors of the DR control strategy within $5 \%$ of $\pm 1 \mathrm{MW} 95 \%$ of the time.

\section{ANALYSIS ON Link Reliability}

\section{A. Outage Probability}

In this paper, outage probability, the probability that the signal-to-noise ratio $^{1}$ (SNR) of the received signal is lower than an outage threshold, is applied to evaluate the reliability of a wireless link. More precisely, let $\gamma$ denote the symbol SNR, and $P_{t}$ be the signal power transmitted from the source node, $\gamma=g P_{t} / N_{0}$. The outage probability, $P_{o}\left(\Gamma_{o}\right)$, is given by

$$
P_{o}\left(\Gamma_{o}\right)=\operatorname{Prob}\left\{\frac{P_{t}}{N_{0}} g \leq \Gamma_{o}\right\},
$$

where $\Gamma_{o}$ is a threshold, called outage SNR.

Note that there are other metrics for communication reliability evaluation, such as bit-error-rate (BER) and packet-errorrate (PER). BER and PER depend on the detailed information of the physical layer techniques such as the modulation and coding. Thus, it is difficult if not impossible to obtain a general expression to relate BER/PER and SNR for any physical layer techniques. The outage probability is more general and independent of the physical layer techniques. Given any physical layer techniques adopted, we can easily map the outage probability to BER and PER.

\footnotetext{
${ }^{1}$ As demonstrated in Section II, a properly designed reservation-based MAC protocol can largely eliminate the interference caused by concurrent communications. Thus, SNR is used here instead of signal-to-interference/noise ratio (SINR).
}

\section{B. Link Reliability}

As demonstrated in Section II, the channel gain depends on the distance between the source and destination. Given the distance $l$, the PDF of SNR, considering both the log-normal shadowing effect (2) and Rayleigh fading (3), is

$$
f_{\Gamma}(\gamma \mid l)=\int_{0}^{\infty} \frac{N_{0}}{P_{t}} f_{G}\left(\frac{N_{0} \gamma}{P_{t}} \mid s\right) \cdot f_{S}(s \mid l) d s .
$$

Thus, the link outage probability based on distance $l$ with outage SNR threshold $\Gamma_{o}$ is

$$
P_{o}\left(\Gamma_{o} \mid l\right)=\int_{0}^{\Gamma_{o}} \int_{0}^{\infty} \frac{N_{0}}{P_{t}} f_{G}\left(\frac{N_{0} \gamma}{P_{t}} \mid s\right) \cdot f_{S}(s \mid l) d s d \gamma .
$$

Therefore, the link reliability can be evaluated by $P_{o}\left(\Gamma_{o}\right)$, which indicates the outage probability for an arbitrary link in a specified network topology setting. Let $v=5 \sqrt{2} / \sigma \log _{10}\left(s / K l^{\epsilon}\right)$,

$$
P_{o}\left(\Gamma_{o}\right)=\int_{-\infty}^{+\infty} \frac{1}{\sqrt{\pi}} e^{-v^{2}} I_{0}\left(\Gamma_{o}, \alpha 10^{\sqrt{2 \pi} v / 10}\right) d v
$$

where

$$
\begin{aligned}
I_{0}\left(\Gamma_{o}, z(v)\right) & =\int_{L^{-}}^{L^{+}}\left(1-e^{-\Gamma_{o} l^{\epsilon} / z(v)}\right) f_{L}(l) d l, \\
z(v) & =\alpha 10^{\sqrt{2 \pi} v / 10}
\end{aligned}
$$

$\alpha=P_{t} K / N_{0}$, and $f_{L}(l)$ is the PDF of the random distance between the source and the destination limited in $\left[L^{-}, L^{+}\right]$.

\section{Approximation of Link Outage Probability}

As in (7)-(8), a double integral is encountered in computing the link outage probability, making it difficult to obtain analytical results and thus compelling us to find a proper approximation.

\section{1) Approximation I}

The link outage probability with the given outage SNR can be approximated using a two-tiered $N$-point Gauss quadrature [15].

For the first tier, Gauss-Legendre quadrature [15] can be applied to compute the inner integral in (8). Thus,

$I_{0}\left(\Gamma_{o}, z(v)\right) \approx \sum_{i=1}^{N} a \omega_{i}^{g l} \cdot f_{L}\left(a x_{i}^{g l}+b\right) \cdot\left(1-e^{-\left(a x_{i}^{g l}+b\right)^{\epsilon} \Gamma_{o} / z(v)}\right)$,

where $a=\left(L^{+}-L^{-}\right) / 2, b=\left(L^{+}+L^{-}\right) / 2, x_{i}^{g l}$ is the $i$-th root of $N$-order Legendre polynomial, and $\omega_{i}^{g l}$ is the weight associated with $x_{i}^{g l}$.

In the second tier, for the integral of normal-weighted function in infinity interval in (7), Gauss-Hermite quadrature can be adopted [15]. Therefore,

$$
P_{o}\left(\Gamma_{o}\right) \approx \sum_{j=1}^{N} \frac{\omega_{j}^{g h}}{\sqrt{\pi}} I_{0}\left(\Gamma_{o}, z\left(x_{j}^{g h}\right)\right),
$$

where $x_{j}^{g h}$ is the $j$-th root of the monic Hermite polynomial, $H_{n}(x)$; its associated weight is given by $\omega_{j}^{g h}=e^{-\left(x_{j}^{g h}\right)^{2}}$. In 
(10) and (11), $g l$ and $g h$ denote the Quadrature method adopted; $x_{i}^{g l}, x_{j}^{g h}, \omega_{i}^{g l}$, and $\omega_{j}^{g h}$ have been tabulated in [15].

2) Approximation II

As shown in [16], the distribution of SNR can be approximated using a single log-normal distribution when $\sigma$ for the shadowing effect is larger than $6 \mathrm{~dB}$. The PDF, shown in (5), can be approximated by

$$
f_{\Gamma}^{\prime}(\gamma \mid l) \approx \frac{\eta}{\sigma_{a} \sqrt{2 \pi} \gamma} \exp \left\{\frac{-\left(10 \log _{10} \gamma-\mu_{a}\right)^{2}}{2 \sigma_{a}^{2}}\right\},
$$

where $\sigma_{a}=\sqrt{\sigma^{2}+5.57^{2}}, \mu_{a}=10 \log _{10}\left(K P_{t} l^{-\epsilon} / N_{0}\right)-\eta C_{e}$, and $C_{e} \approx 0.57721566$ is the Euler's constant.

In this case, the outage probability can be derived using a one-step approximation applying Gauss-Legendre quadrature. Therefore,

$$
\begin{aligned}
P_{o}\left(\Gamma_{o}\right) & =\int_{0}^{\Gamma_{o}} \int_{L^{-}}^{L^{+}} f_{\Gamma}^{\prime}(\gamma \mid l) \cdot f_{L}(l) d l d \gamma \\
& \approx \sum_{i=1}^{N} \omega_{i}^{g l} g_{2}^{g l}\left(\Gamma_{o}, x_{i}^{g l}\right)
\end{aligned}
$$

where

$$
\begin{aligned}
g_{2}^{g l}\left(\Gamma_{o}, x_{i}^{g l}\right) & =\frac{a f_{L}(u)}{2} \cdot \operatorname{erfc}\left[\frac{1}{\sqrt{2} \sigma_{a}} Y\left(\Gamma_{o}, u\right)\right], \\
Y\left(\Gamma_{o}, u\right) & =10 \log _{10}\left(\frac{\Gamma_{o}}{\alpha u^{\epsilon}}\right)+C_{e} \eta \\
u & =a x_{i}^{g l}+b
\end{aligned}
$$

and $\operatorname{erfc}(\cdot)$ is the complementary error function.

The proofs of (10), (11), and (13) can be found in Appendix A.

\section{Analysis on Network-Level Reliability}

In this section, we discuss the network-level reliability with a given number of smart meters, and study the impact of network topology on reliability.

To apply a link reliability model above for network-level reliability, the outage SNR threshold $\Gamma_{o}$ needs be set according to the required reliability, i.e., BER $\leq 10^{-5}$ and the physical techniques, i.e., BPSK/MQAM modulation. $\Gamma_{o}$ can be acquired using Monte Carlo simulation or a two-state Markov model, which has been proposed in the literature to characterize the behavior of packet errors in fading channels for a wide range of parameters [17].

\section{A. Reliability in a Single-Hop Network}

In a single-hop network, all smart meters are directly connected to the DA, as shown in Fig. 1(a). Assuming all $N_{s}$ smart meters are distributed uniformly and independently, the packet delivery ratio, as the performance index of network-level reliability, can be modelled as a Bernoulli process with parameter $p=1-P_{o}\left(\Gamma_{o}\right)$, which indicates the probability of successful delivery between a smart meter and the DA. Let $P_{s}^{1 h}(\theta)$ denote the probability that packet delivery ratio is no less than $\theta$, i.e., at least $\left[\theta N_{s}\right\rceil$ packets are successfully delivered to their destinations $(0 \leq \theta \leq 1)$. Therefore,

$$
P_{s}^{1 h}(\theta)=\sum_{i=0}^{\left\lfloor(1-\theta) N_{s}\right\rfloor}\left(\begin{array}{c}
N_{s} \\
i
\end{array}\right) P_{o}\left(\Gamma_{o}\right)^{i}\left(1-P_{o}\left(\Gamma_{o}\right)\right)^{N_{s}-i} .
$$

Note that the accuracy of $P_{s}^{1 h}(\theta)$ is related to $f_{L}(\cdot)$, the PDF of the distance between a smart meter and the DA, and the distance distribution depends on the shape of the coverage area. Typically, if an omni-directional antenna is used, the shape can be approximated as a circle with the DA at the center. However, if multiple DAs are used to cover a large area, a hexagon shape is more accurate than a circle for computing the random distance [14].

\section{B. Reliability in a Multi-Hop Network}

Unlike a single-hop network, in a multi-hop network a packet may be relayed by other smart meters or relays [1] before it arrives at the destination. For an arbitrary smart meter, the multi-hop networks' end-to-end outage probability in sending or receiving a correct packet to or from the DA is determined by two factors: the number of hops along its packet routing path and the outage probability for each hop.

Given an $m$-hop routing path between a smart meter and the DA, it means that there are $(m-1)$ other smart meters along the routing path to forward the packet. Let $l_{k}$ denote the distance of the $k$-th hop along the routing path, and $P_{o}^{(m)}\left(\Gamma_{o}\right)$ denote the end-to-end outage probability with outage SNR threshold $\Gamma_{o}$,

$$
P_{o}^{(m)}\left(\Gamma_{o}\right)=1-\prod_{k=1}^{m} \int_{L^{-}}^{L^{+}}\left[1-P_{o}\left(\Gamma_{o} \mid l_{k}\right)\right] f_{L}\left(l_{k}\right) d l_{k}
$$

where $P_{o}\left(\Gamma_{o} \mid l_{k}\right)$ is the link outage probability determined by (6).

In a multi-hop network, the number of hops needed to deliver a packet between a smart meter and the DA depends on the network topology and the adopted routing algorithm. In this paper, we study the clustering-based grid topology, ${ }^{2}$ as shown in Fig. 1(b) and Manhattan routing scheme [13]. Assuming that a large $E \times E$ area is covered using square-clusters with an edge length of $R$, there can be $(2 M+1)^{2}$ clusters, where $M=$ $\lceil(E-R) / 2 R\rceil$. Let $P_{h}(m)$ denote the probability of a smart meter taking $m$ hops to reach the DA,

$$
P_{h}(m)= \begin{cases}\frac{1}{(2 M+1)^{2}}, & m=1 \\ \frac{4(m-1)}{(2 M+1)^{2}}, & m=2,3, \ldots, M+1 \\ \frac{4(2 M+2-j)}{(2 M+1)^{2}}, & m=M+2, \ldots, 2 M+1 .\end{cases}
$$

Let $P_{s}^{m h}(\theta)$ denote the probability that the packet deliver ratio is at least $\theta$ in a multi-hop network. Therefore, $P_{s}^{m h}(\theta)$ in a $E \times E$ multi-hop cluster-based network with unit grid size $R \times R$ grid is

$$
P_{s}^{m h}(\theta)=\sum_{i=0}^{\left\lfloor(1-\theta) N_{s}\right\rfloor}\left(\begin{array}{c}
N_{s} \\
i
\end{array}\right)\left(1-P_{s}\left(\Gamma_{o}\right)\right)^{i} P_{s}\left(\Gamma_{o}\right)^{N_{s}-i},
$$

\footnotetext{
${ }^{2}$ The cluster-header selection algorithm has been investigated extensively in the literature and is beyond the scope of this paper.
} 


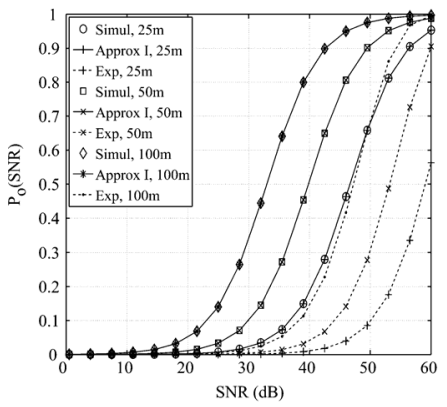

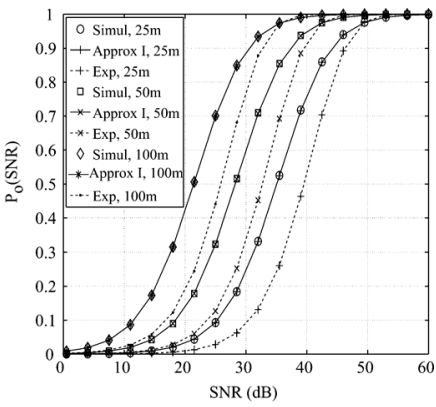

(b)
Fig. 4. Link outage probability Approximation I. (a) In a circle. (b) In two parallel squares.

where $P_{s}\left(\Gamma_{o}\right)=\sum_{m=1}^{2 M+1} P_{h}(m)\left[1-P_{o}^{(m)}\left(\Gamma_{o}\right)\right]$. In addition, note that the link distance distributions of the first, last, and other hops can be different in the above network topology (see Appendix B).

\section{Model VAlidation AND APPLICATIONS}

In this section, we discuss extensive simulations conducted to evaluate the accuracy of the proposed reliability model of communication networks at both link and network levels. In addition, as an application of the model developed, the maximum coverage of a DA is obtained with different reliability levels, and comparison is presented between using the single-hop and multi-hop network topologies. We use the following channel parameters on all links between smart meters and the DA: $P_{t}=$ $1 \mathrm{~mW}$, the standard deviation for the log-normal shadowing effect, $\sigma=3 \mathrm{~dB}$, the path loss exponent, $\epsilon=2.27$, and the path loss constant, $K=46.4 \mathrm{~dB}$ (for $2.4 \mathrm{GHz}$ carrier frequency) [18].

\section{A. Model Validation}

The accuracy of the link outage probability model is tested by comparing it to the Monte Carlo simulation results. The random distance distributions (see Appendix B) in the two types of topologies are adopted. One is a circle, which fits to the wireless communication link between a smart meter and the DA in the single-hop communication architecture; and the other is two parallel squares, which fits to the link between two cluster-header smart meters in multi-hop networks.

Fig. 4 shows $P_{o}\left(\Gamma_{o}\right)$ computed using Approximation I (11) with various circle radii or square edges as 25,50 , and 100 meters. In all cases, the results of our analysis match well with the simulation results. Results of a third analysis approximation are also presented, in which, for simplification, the average link distance is used instead of the random distance distribution, and only the random effects of the shadowing effect and Rayleigh fading are considered. As Fig. 4 shows, it is obvious that the method using the average distance significantly underestimates the link outage probability, which can cause unacceptable overestimation of the link reliability.

In Fig. 5, the accuracy of two approximation methods, I and II, are compared with different standard derivations of shadowing effect, $\sigma_{1}=3 \mathrm{~dB}$ and $\sigma_{2}=8 \mathrm{~dB}$. It can be found the SNR distribution computed by Approximation II becomes close

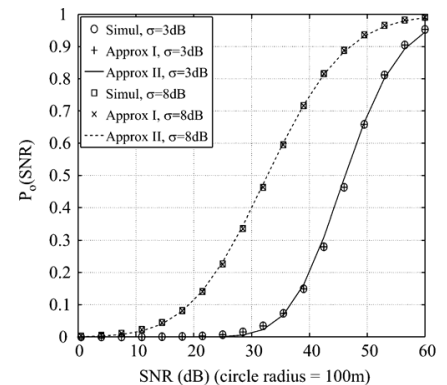

(a)

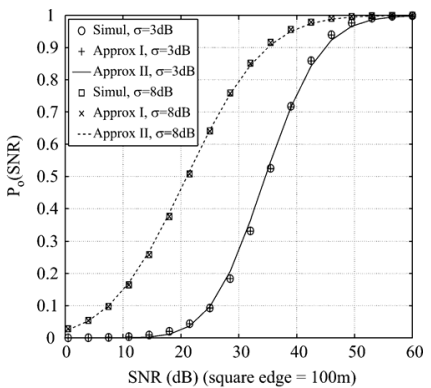

(b)
Fig. 5. Link outage probability Approximation II. (a) In a circle. (b) In two parallel squares.

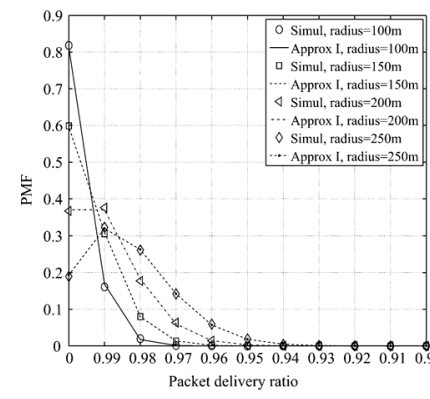

(a)

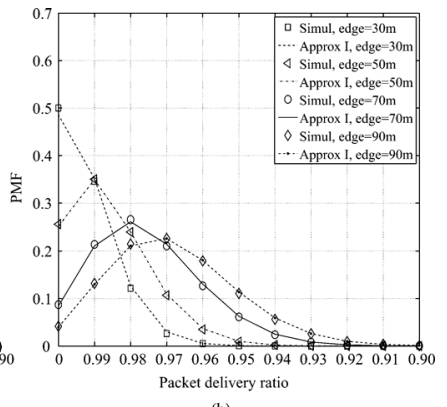

(b)
Fig. 6. PMF of packet delivery ratio. (a) In a single-hop network. (b) In a multi-hop network.

to the results in Monte Carlo simulations when $\sigma$ is larger than $6 \mathrm{~dB}$.

The network level reliability model is verified in Fig. 6, which shows the probability mass function (PMF) of the packet delivery ratio given the outage SNR, $\Gamma_{o}=6 \mathrm{~dB}$. With the single-hop architecture [Fig. 6(a)], as the coverage area is enlarged, the distance between a smart meter and the DA also increases, so that the peak value of the PMF curve shifts low and right to the low packet delivery ratio region.

With the multi-hop architecture, the setting is slightly different from the single-hop scenario in that the coverage area is fixed at $1 \times 1 \mathrm{~km}^{2}$ but the square size is increased. In Fig. $6(\mathrm{~b})$, the PMF of packet delivery ratio in a multi-hop network shows the same trend as that in single-hop network. Although the number of hops is reduced with an increased cluster size, the packet delivery ratio is more sensitive to the communication distance, as path loss increases much faster as a function of powers of the distance.

\section{B. Model Application-Maximum Coverage}

To explore the maximum coverage that a DA can provide when the delivery ratio is guaranteed, search algorithms [19] can be developed by applying the proposed reliability indexes. In the following, a one-dimensional search algorithm is used to find the maximum diameter in the single-hop scenario, and a two-dimensional search algorithm is adopted for the maximum coverage edge length and the optimal cluster size in the multi-hop scenario.

Recalling the results shown in Section III, up to $4 \%$ delivery failure ratio is acceptable for the DR control. Fig. 7 shows the maximum coverage, $L^{+}$, in which the four groups of bars represent the maximum coverages with different outage SNR thresholds. For each bar group, the height of the bars indicates the 


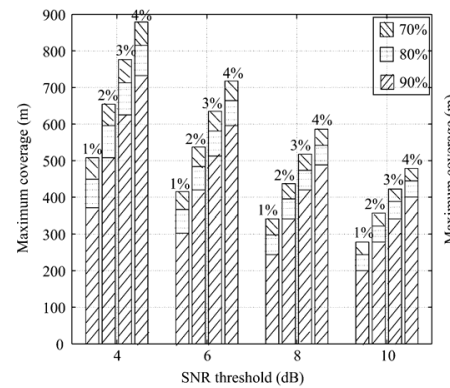

(a)

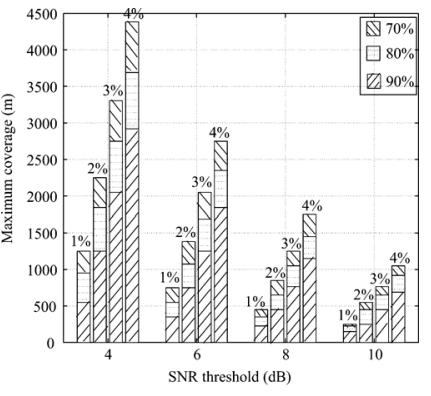

(b)
Fig. 7. Maximum coverage. (a) In a single-hop network. (b) In a multi-hop network.

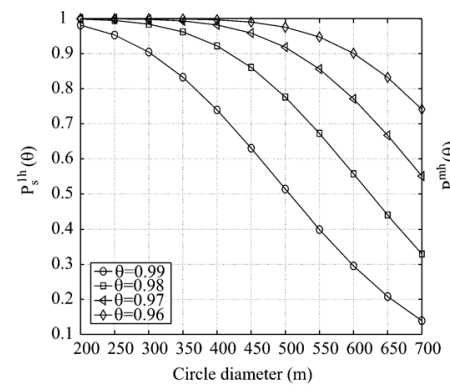

(a)

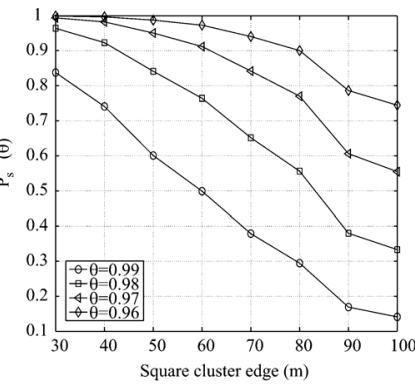

(b)
Fig. 8. Packet delivery ratio vs. network size. (a) In a single-hop network. (b) In a multi-hop network.

maximum coverage ensuring that the link outage probability is lower than $1 \%, 2 \%, 3 \%$, and $4 \%$ with packet delivery ratio no less than $70 \%, 80 \%$, and $90 \%$.

Another important observation in Section III is that the DR performance is more vulnerable to delivery ratio disproportion among different groups of users. Results in Fig. 8 demonstrates that such disproportion exists in the communication networks if the same physical layer techniques are adopted, such as modulation and coding, etc.; it is found that the probability of packet delivery ratio degrades quickly w.r.t. the distance in both single-hop and multi-hop networks. Due to the path-loss between smart meters and the shadowing effect, as the coverage increases, the signals from smart meters in the edges are typically weaker. Thus communication services would be far worse for the smart meters at the edges of the coverage area. To design reliable communication networks for smart grid, extra protection for edge smart meters should be considered, such as re-transmissions in the MAC layer or adaptive modulation/coding in the physical layer.

\section{CONCLUSION}

In this paper, we have modelled and analyzed the reliability of wireless communication services for the smart grid. We have first investigated the impact of communication losses on DR control accuracy. Model-based simulations reveal the importance of communication service reliability for effective DR control. Next, we have modelled communication reliability and evaluated it in the link level, considering the log-normal shadowing effect, Rayleigh fading, and random locations of smart meters. Extended from the link level model, communication reliability in both single-hop and multi-hop wireless networks has also been modelled. Note that the communication model proposed is applicable for a general DR control strategy, not limited to the specific one in [4]. Monte Carlo simulations were conducted to verify the accuracy of the proposed model. The proposed models have been applied to quantify the maximum coverage of a wireless network with the reliability requirements.

More research efforts are beckoned to fully understand the interaction of communication network design and DR control in a smart grid. One issue is to provide similar reliability for all smart meters, such as retransmission in the MAC layer and adaptive modulation/coding (AMC) in the physical layer. Our model can be extended to consider retransmission by computing the failure probability of all (re-)transmissions, and consider AMC by setting an appropriate SNR threshold according to the physical techniques. Note that when retransmission is adopted to provide more reliable information delivery, it raises a new issue of optimizing MAC protocol and resource allocation using hybrid contention- and reservation-based strategies. Another issue is the impact of network topology and routing algorithms on communication reliability. To explore the optimal topology or routing algorithm, our model can be applied for evaluating different network topologies and routing algorithms by modifying the distribution of the communication distance $\left(f_{L}(\cdot)\right)$ and number of hops $\left(P_{h}(\cdot)\right)$ between the smart meters and DA accordingly.

\section{APPENDIX A}

\section{Proof of Link OUtAge Probability ApProximations}

\section{A.1. Equation (10) in Approximation I}

The Gauss-Legendre quadrature [15] can be used to calculate integral of $f(x)$ within $[-1,1]$, that

$$
\int_{-1}^{1} f(x) d x=\sum_{i=1}^{N} \omega_{i}^{g l} f\left(x_{i}^{g l}\right) .
$$

Let $f(x)=\left(1-e^{-\Gamma_{o} x^{\epsilon} / z(v)}\right) f_{L}(x)$, for an integral interval $\left[L^{-}, L^{+}\right]$,

$$
\begin{aligned}
\int_{L^{-}}^{L^{+}} & f(x) d x \\
& =\frac{L^{+}-L^{-}}{2} \int_{-1}^{1} f\left(\frac{L^{+}-L^{-}}{2} x+\frac{L^{+}+L^{-}}{2}\right) d x .
\end{aligned}
$$

Thus, (10) can be derived by substituting (18) into (19). A.2. Equation (11) in Approximation I

The Gauss-Hermite quadrature [15] can be used to calculate infinite integral of normal-weight $f(x)$ as follows:

$$
\int_{-\infty}^{+\infty} e^{-x^{2}} f(x) d x=\sum_{j=1}^{N} \omega_{j}^{g h} f\left(x_{j}^{g h}\right) .
$$

Therefore, (11) can be obtained by applying (20) with $f(x)=1 / \sqrt{\pi} I_{0}\left(\Gamma_{o}, z(x)\right)$.

\section{A.3. Equation (13) in Approximation II}

In (12), let $\gamma^{\prime}=10 \log _{10} \gamma-\mu_{a}$ and $\gamma_{M}^{\prime}\left(\Gamma_{o}, l\right)=$ $10 \log _{10} \Gamma_{o}-10 \log _{10}\left(K P_{t} l^{-\epsilon} / N_{0}\right)+\eta C_{e}$; thus,

$$
\begin{aligned}
& f_{\Gamma}(\gamma \mid l) d \gamma=\frac{1}{\sigma_{a} \sqrt{2 \pi}} \\
& \times \exp \left(\frac{-\gamma^{\prime 2}}{2 \sigma_{a}^{2}}\right) d \gamma^{\prime}, \gamma^{\prime} \in\left(-\infty, \gamma_{M}^{\prime}\left(\Gamma_{o}, l\right)\right],
\end{aligned}
$$


and then,

$$
\begin{aligned}
P_{o}\left(\Gamma_{o}\right)= & \int_{L^{-}}^{L^{+}} f_{L}(l) d l \int_{-\infty}^{\gamma_{M}^{\prime}\left(\Gamma_{o}, l\right)} \frac{1}{\sigma_{a} \sqrt{2 \pi}} \\
& \times \exp \left(\frac{-\gamma^{\prime 2}}{2 \sigma_{a}^{2}}\right) d \gamma^{\prime} \\
= & \int_{L^{-}}^{L^{+}} f_{L}(l) \cdot \frac{1}{2} \operatorname{erfc}\left(\frac{\gamma_{M}^{\prime}\left(\Gamma_{o}, l\right)}{\sqrt{2} \sigma_{a}}\right) d l .
\end{aligned}
$$

Similar to the proof of (10), (13) can be obtained by applying Gauss-Legendre quadrature to calculate (22).

\section{APPENDIX B \\ RANDOM Distance DistRiBution}

The PDF of random distance between two points in a circle, between two points in a square, and between two points in two parallel squares can be found in [20].

It is easy to get the PDF of random distance from the centric to another point in the neighbouring square with edge length $R$ as shown in (23).

$$
f_{L}(l)= \begin{cases}\frac{2 l}{R^{2}} \cos ^{-1}\left(\frac{R}{2 l}\right) & \frac{1}{2} R \leq l<\frac{\sqrt{2}}{2} R \\ \frac{2 l}{R^{2}} \sin ^{-1}\left(\frac{R}{2 l}\right) & \frac{\sqrt{2}}{2} R \leq l<\frac{3}{2} R \\ \frac{2 l}{R^{2}}\left[\sin ^{-1}\left(\frac{R}{2 l}\right)-\cos ^{-1}\left(\frac{3 R}{2 l}\right)\right] & \frac{3}{2} R \leq l \leq \frac{\sqrt{10}}{2} R .\end{cases}
$$

\section{REFERENCES}

[1] W. Luan, D. Sharp, and S. Lancashire, "Smart grid communication network capacity planning for power utilities," in Proc. IEEE PES Transm. Distrib. Conf. Expo., Apr. 2010, pp. 1-4.

[2] D. Callaway, "Tapping the energy storage potential in electric loads to deliver load following and regulation with application to wind energy," Energy Convers. Manag., vol. 50, no. 9, pp. 1389-1400, 2009.

[3] S. Parkinson, D. Wang, C. Crawford, and N. Djilali, "Comfort-constrained distributed heat pump management," in Proc. Int. Conf. Smart Grid Clean Energy Technol. (ICSGCE), 2011, vol. 12, pp. 849-855.

[4] N. Lu, "An evaluation of the HVAC load potential for providing load balancing service," IEEE Trans. Smart Grid, vol. 3, no. 2, pp. 1263-1270, Sep. 2012.

[5] Z. Fadlullah, N. Kato, R. Lu, X. Shen, and Y. Nozaki, "Toward secure targeted broadcast in smart grid," IEEE Commun. Mag., vol. 50, no. 5 , pp. 150-156, May 2012.

[6] R. Deng, S. Maharjan, X. Cao, J. Chen, Y. Zhang, and S. Gjessing, "Sensing-delay tradeoff for communication in cognitive radio enabled smart grid," in Proc. IEEE SmartGridComm, Brussels, Belgium, Oct. 2011, pp. $155-160$.

[7] M. Fouda, Z. Fadlullah, N. Kato, R. Lu, and X. Shen, "A lightweight message authentication scheme for smart grid communications," IEEE Trans. Smart Grid, vol. 2, no. 4, pp. 675-685, Dec. 2011.

[8] A. Kashyap and D. Callaway, "Controlling distributed energy constrained resources for power system ancillary services," in Proc. IEEE PMAPS, 2010, pp. 407-412.

[9] D. Niyato, P. Wang, E. Hossain, and Z. Han, "Impact of packet loss on power demand estimation and power supply cost in smart grid," in Proc. IEEE WCNC, 2011, pp. 6-10.

[10] P. Bhagwat, P. Mishra, and S. Tripathi, "Effect of topology on performance of reliable multicast communication," in Proc. IEEE INFOCOM, Jun. 1994, vol. 2, pp. 602-609.

[11] M. Ghaderi, D. Towsley, and J. Kurose, "Reliability gain of network coding in lossy wireless networks," in Proc. IEEE INFOCOM, Apr. 2008, pp. 2171-2179.
[12] L. Zheng, S. Parkinson, D. Wang, L. Cai, and C. Crawford, "Energy efficient communication networks design for demand response in smart grid," in Proc. Int. Conf. Wireless Commun. Signal Process. (WCSP), Nov. 2011, pp. 1-6.

[13] Y.Xu, J. Heidemann, and D. Estrin, "Geography-informed energy conservation for ad hoc routing," in Proc. ACM MOBICOM, 2001, pp. $70-84$.

[14] Y. Zhuang, Y. Luo, L. Cai, and J. Pan, "A geometric probability model for capacity analysis and interference estimation in wireless mobile cellular systems," Proc. IEEE GLOBECOM, Dec. 2011.

[15] F. Olver, D. Lozier, R. Boisvert, and C. Clark, NIST Handbook of Mathematical Functions. New York: Cambridge Univ. Press, 2010.

[16] A. Turkmani, "Probability of error for m-branch macroscopic selection diversity," IEE Proc. I, Commun., Speech, Vis. , vol. 139, no. 1, pp. 71-78, Feb. 1992.

[17] M. Zorzi, R. Rao, and L. Milstein, "Arq error control for fading mobile radio channels," IEEE Trans. Veh. Technol., vol. 46, no. 2, pp. 445-455, May 1997

[18] IST-4-027756 WINNER II D 1.1.2 v1.2, WINNER II Channel Models, 2006.

[19] A. Antoniou and W. Lu, Practical Optimization: Algorithms and Engineering Applications. New York: Springer, 2007.

[20] Y. Zhuang, J. Pan, and L. Cai, "Minimizing energy consumption with probabilistic distance models in wireless sensor networks," in Proc. IEEE INFOCOM, Mar. 2010, pp. 1-9.

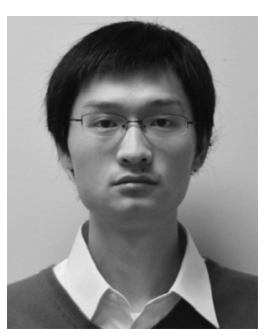

Lei Zheng (S'11) received the B.S. and M.S. degrees in electrical engineering from Beijing University of Posts and Telecommunications, Beijing, China, in 2007 and 2010, respectively. He is currently working toward a Ph.D. degree with the Department of Electrical and Computer Engineering, University of Victoria, Victoria, BC, Canada. His research interest is medium access control and resource management in wireless networks and smart grid.

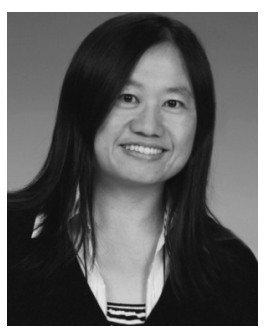

Ning Lu (M'98-SM'05) received her B.S.E.E. degree from Harbin Institute of Technology, Harbin, China, in 1993, and her M.S. and Ph.D. degrees in electric power engineering from Rensselaer Polytechnic Institute, Troy, NY, in 1999 and 2002, respectively.

She is an Associate Professor in electrical and computer engineering at North Carolina State University, Raleigh. She was a Senior Research Engineer with the Energy and Environment Directorate, Pacific Northwest National Laboratory, Richland, WA. Her research interests are in modeling and analyzing power system load behaviors with a focus on the smart grid technology implementation on power system distribution grids.

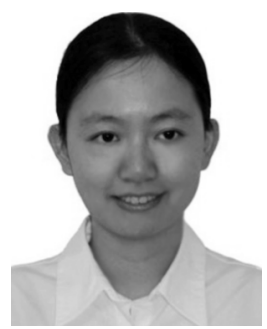

Lin Cai (S'00-M'06-SM'10) received the M.A.Sc. and $\mathrm{Ph} . \mathrm{D}$. degrees in electrical and computer engineering from the University of Waterloo, Waterloo, ON, Canada, in 2002 and 2005, respectively.

Since 2005, she has been an Assistant Professor and then an Associate Professor with the Department of Electrical and Computer Engineering, University of Victoria, Victoria, BC, Canada. Her research interests include wireless communications and networking, with a focus on network protocol and architecture design supporting emerging multimedia traffic over wireless, mobile, ad-hoc, and sensor networks. She has been an Associate Editor for IEEE TRANSACTIONS ON WIRELESS COMMUNICATIONS, IEEE TRANSACTIONS ON Vehicular TEChNOLOgY, EURASIP Journal on Wireless Communications and Networking, the International Journal of Sensor Networks, and the Journal of Communications and Networks. 